\title{
Critical earthquake load inputs for multi-degree-of-freedom inelastic structures
}

\author{
Abbas Moustafa \\ Department of Civil Engineering, Minia University, Minia 61111, Egypt \\ Department of Civil Engineering, Nagasaki University, Nagasaki 852-8521, Japan
}

\begin{abstract}
The problem of modeling earthquake ground motions as design inputs for multi-degree-of-freedom inelastic structures is studied. The earthquake acceleration is expressed as a Fourier series modulated by an envelope function. The coefficients of the series representation are calculated such that the structure inelastic deformation is maximized subjected to predefined constraints. These constraints are taken to reflect known characteristics of recorded earthquakes such as upper bounds on the energy and peak values of the ground acceleration, velocity and displacement, and upper and lower limits on the Fourier spectra of the ground acceleration. The material stress-strain behavior is modeled using bilinear and elastic-plastic relations. The resulting nonlinear optimization problem is solved by using the sequential quadratic optimization method. Issues related to various forms of energy dissipated by the inelastic structure are explored. The study also examines the effect of nonlinear damping models and the influence of the strain hardening ratio (ratio of the post-yield stiffness to the pre-yield stiffness) on the derived optimal earthquake and associated inelastic deformation. The formulation is demonstrated for a two-story inelastic building frame with nonlinear damping.
\end{abstract}

Keywords: earthquake loads; inelastic buildings; ductility ratio; strain hardening ratio; hysteretic energy; nonlinear damping; nonlinear optimization.

\section{Introduction}

The earthquake loading in a given region is related to its probable intensity and likelihood of occurrence which, in turn, depends on the region seismicity. Earthquake hazard and regional seismicity, in turn, are related to the seismotectonics or plate tectonics of that region. Heat loss from the earth interior drives the plate tectonic engine, forcing more than a dozen large rigid plates to move around the earth surface, grinding past each other, forming earthquakes. The study of seismotectonics is in general concerned with understanding what controls surface deformation that produces earthquakes. Specifically, seismotectonics studies deal with understanding the distribution of earthquakes in space, time, and size. The study of seismotectonics is beyond the scope of this paper, and, further details can be found in [1,2]. The framework adopted in this paper for modeling earthquake loads for inelastic structures belongs to the class of engineering models that aim to replicate gross features of recorded ground motions.

The modeling of earthquake ground motions as design inputs for engineering structures has received significant research attention worldwide for the last five decades or so [3]. However, the high level of uncertainty associated with the earthquake phenomenon and severe damages caused by strong motion earthquakes (e.g., San Fernando 1971, Hyogoken-Nanbu 1995 and the more recent 2008 China earthquake) pose a significant challenge for structural engineers and researchers to mitigate the disaster and risk caused by earthquake ground motions. It is, thus, essential to develop robust methods for seismic-resistant design of structures. The specification of accurate and reliable design earthquake loads that are representative of the destructive potential of the ground motion at a given site is the first step towards achieving this goal. Earthquake loads are specified as design inputs for structures in terms of design or hazard response spectra of the site, the time history 
of the ground acceleration or by using the method of random vibrations. The method of seismic critical excitation has been established during the last four decades as a counterpart to these methods. In this method, the worst possible future earthquake is derived by using inverse dynamic analysis and optimization techniques such that the structure performance is minimized, and at the same time, the earthquake ground motion satisfies known features of recorded earthquakes. Moustafa [4] and Takewaki [5] provided a comprehensive review on this subject. This method relies on the fact that, for many parts of the world, available data on strong ground motions is either inhomogeneous or insufficient. The method relies also on the high level of uncertainty associated with earthquake occurrence and on the uncompromised safety of lifeline and important structures (e.g., nuclear power plants, electric power, gas and water networks, chemicals and water storage tanks).

As is well known, the inclusion of material nonlinearity in earthquake-resistant design of structures is of central importance in earthquake engineering. It is, thus, of interest to investigate the development of this method to structures deforming into the inelastic stage. This is particularly true when dealing with response analysis of structures driven by extreme loads as is the case with critical earthquake loads.

While the modeling of critical earthquake loads for linear structures has been widely studied, the problem of modeling critical earthquake excitations for nonlinear structures has been studied to a very limited extent in the existing literature. Iyengar [6] derived critical earthquake inputs for Duffing oscillators under a constraint on the input energy. Drenick [7] showed that the critical excitation for a nonlinear system is the impulse response function of the linearized system reversed in time. Philippacopoulos and Wang [8] established critical inelastic response spectra using past recorded accelerograms as basis functions for the critical input. The modeling of critical excitations for elastic-plastic and hysteretic single-degree-of-freedom (SDOF) systems using calculus of variations was carried out by Westermo [9]. He showed that the critical inputs for linear systems are harmonic while those for inelastic systems are not harmonic. The critical excitations computed in these studies, however, do not possess realistic characteristics of recorded ground motions, and, thus are not realistic models for earthquake loads. Pirasteh et al., [10] modeled critical seismic inputs for inelastic frame structures by maximizing an approximate function for the inelastic energy of the structure. Recently, Takewaki $[11,12]$ developed critical power spectral density functions for SDOF and multi-degree-of-freedom (MDOF) elastic-plastic buildings using statistical linearization. Abbas and Manohar $[13,14]$ developed a reliability-based framework for computing random critical earthquake loads for nonlinear and parametrically excited structures by maximizing the structure's failure probability or the reliability index. This approach combines methods of structural reliability analysis, response surface modeling and nonlinear programming in computing seismic inputs for structures having cubic force-displacement relations. More recently, Abbas [15] derived critical earthquake inputs for SDOF elastic-plastic structures by maximizing the ductility ratio.

This paper extends the previous study by this author [15] on modeling critical earthquake loads for SDOF elastic-plastic structures to MDOF elastic-plastic and bilinear structures. The study also explores issues related to energy dissipated by inelastic structures. The earthquake load is expressed in terms of a Fourier series modulated by an envelope function. These coefficients are computed such that the structure inelastic deformation is maximized subjected to predefined constraints. These constraints include upper bounds on the earthquake energy and peak values of ground acceleration, velocity and displacement and upper and lower limits on the Fourier spectra of the ground acceleration. The resulting nonlinear optimization problem is solved by using the sequential quadratic optimization method. Numerical illustrations on modeling critical earthquakes for two-story inelastic building with hysteretic damping are provided. Section 2 demonstrates the dynamic analysis for MDOF inelastic structures. The formulation for modeling critical earthquake inputs for inelastic structures is developed in Section 3 and various energy forms dissipated by 
1 inelastic structures are derived in Section 4. The proposed formulation is demonstrated for a

2 two-story frame building in Section 5. \\ 2. Dynamic analysis of MDOF inelastic structures under earthquake ground motion}

The equation of motion for an $N$-nonlinear MDOF structure driven by a single component of earthquake acceleration $\ddot{x}_{g}(t)$ is given by $[1,16,17]$ :

$$
\mathbf{M} \ddot{\mathbf{X}}(t)+\mathbf{C} \dot{\mathbf{X}}(t)+\mathbf{F}_{s}(t)=\mathbf{P}(t)=-\mathbf{M}\{\mathbf{1}\} \ddot{x}_{g}(t)
$$

where, $\mathbf{M}, \mathbf{C}$, are the mass and damping matrices of the structure, respectively, $\mathbf{F}_{s}(t)$ is the vector of hysteretic restoring forces, $\{\mathbf{1}\}$ is a vector of ones, $\mathbf{X}(t)$ is the structure displacement vector and dot indicates differentiation with respect to time. Note that, for nonlinear damping models, the damping matrix $\mathbf{C}$ is a function of the deformed shape of the structure. Figure 1 depicts the relationship between the deformation and the hysteretic restoring force for bilinear and elastic-plastic materials. The incremental form of equation (1) can be written as:

$$
\mathbf{M} \Delta \ddot{\mathbf{X}}+\mathbf{C} \Delta \dot{\mathbf{X}}+\mathbf{K}_{s} \Delta \mathbf{X}=\Delta \mathbf{P}=-\mathbf{M}\{\mathbf{1}\}\left[\ddot{x}_{g}\left(t_{k+1}\right)-\ddot{x}_{g}\left(t_{k}\right)\right]
$$

where

$$
\Delta \ddot{\mathbf{X}}=\ddot{\mathbf{X}}\left(t_{k+1}\right)-\ddot{\mathbf{X}}\left(t_{k}\right) ; \Delta \dot{\mathbf{X}}=\dot{\mathbf{X}}\left(t_{k+1}\right)-\dot{\mathbf{X}}\left(t_{k}\right) ; \Delta \mathbf{X}=\mathbf{X}\left(t_{k+1}\right)-\mathbf{X}\left(t_{k}\right)
$$

and $\mathbf{K}_{s}$ is the stiffness corresponding to the displacement from $\mathbf{X}\left(t_{k}\right)$ to $\mathbf{X}\left(t_{k+1}\right)$. We consider the case where the solution for the response is obtained by using the Newmark $\beta$-method. Accordingly, the velocity and displacement responses at time $t_{k+1}$ are given as:

$$
\dot{\mathbf{X}}_{k+1}=\dot{\mathbf{X}}_{k}+(1-\delta) \ddot{\mathbf{X}}_{k} \Delta t+\delta \ddot{\mathbf{X}}_{k+1} \Delta t
$$

$$
\mathbf{X}_{k+1}=\mathbf{X}_{k}+\dot{\mathbf{X}}_{k} \Delta t+\left(\frac{1}{2}-\alpha\right) \ddot{\mathbf{X}}_{k}(\Delta t)^{2}+\alpha \ddot{\mathbf{X}}_{k+1}(\Delta t)^{2}
$$

where $\delta$ and $\alpha$ are the parameters of the Newmark $\beta$-method and $\Delta t=t_{k+1}-t_{k}$ is the time step. Equations (4) can be written in an incremental form as:

$$
\begin{aligned}
& \Delta \dot{\mathbf{X}}=(1-\delta) \ddot{\mathbf{X}}_{k} \Delta t+\delta \ddot{\mathbf{X}}_{k+1} \Delta t \\
& \Delta \mathbf{X}=\dot{\mathbf{X}}_{k} \Delta t+\left(\frac{1}{2}-\alpha\right) \ddot{\mathbf{X}}_{k}(\Delta t)^{2}+\alpha \ddot{\mathbf{X}}_{k+1}(\Delta t)^{2}
\end{aligned}
$$

Substituting equations (5) into equation (2) we get:

$$
\mathbf{M} \Delta \ddot{\mathbf{X}}+\mathbf{C}\left[(1-\delta) \ddot{\mathbf{X}}_{k} \Delta t+\delta \ddot{\mathbf{X}}_{k+1} \Delta t\right]+\mathbf{K}_{s}\left[\dot{\mathbf{X}}_{k} \Delta t+\left(\frac{1}{2}-\alpha\right) \ddot{\mathbf{X}}_{k}(\Delta t)^{2}+\alpha \ddot{\mathbf{X}}_{k+1}(\Delta t)^{2}\right]=\Delta \mathbf{P}
$$

Substituting $\ddot{\mathbf{X}}_{k+1}=\ddot{\mathbf{X}}_{k}+\Delta \ddot{\mathbf{X}}$ in the above equation leads to:

$$
\mathbf{M} \Delta \ddot{\mathbf{X}}+(1-\delta) \Delta t \mathbf{C} \ddot{\mathbf{X}}_{k}+\delta \Delta t \mathbf{C} \ddot{\mathbf{X}}_{k}+\delta \Delta t \mathbf{C} \Delta \ddot{\mathbf{X}}+\Delta t \mathbf{K}_{s} \dot{\mathbf{X}}_{k}+\left(\frac{1}{2}-\alpha\right)(\Delta t)^{2} \mathbf{K}_{s} \ddot{\mathbf{X}}_{k}
$$

$$
+\alpha(\Delta t)^{2} \mathbf{K}_{s} \ddot{\mathbf{X}}_{k}+\alpha(\Delta t)^{2} \mathbf{K}_{s} \Delta \ddot{\mathbf{X}}=\Delta \mathbf{P}
$$

Collecting similar terms and simplifying, it follows that:

$$
\left[\mathbf{M}+\delta \Delta t \mathbf{C}+\alpha(\Delta t)^{2} \mathbf{K} s\right] \Delta \ddot{\mathbf{X}}+\Delta t \ddot{\mathbf{X}}_{k}+\Delta t \mathbf{K}_{s} \dot{\mathbf{X}}_{k}+\frac{1}{2}(\Delta t)^{2} \mathbf{K}_{s} \ddot{\mathbf{X}}_{k}=\Delta \mathbf{P}
$$

Solving for $\Delta \ddot{\mathbf{X}}$ we get:

$$
\Delta \ddot{\mathbf{X}}=\left[\mathbf{M}+\delta \Delta t \mathbf{C}+\alpha(\Delta t)^{2} \mathbf{K}_{s}\right]^{-1}\left[\Delta \mathbf{P}-\Delta t \ddot{\mathbf{X}}_{k}-\Delta t \mathbf{K}_{s} \dot{\mathbf{X}}_{k}-\frac{1}{2}(\Delta t)^{2} \mathbf{K}_{s} \ddot{\mathbf{X}}_{k}\right]
$$

Equation (9) provides the solution for $\Delta \ddot{\mathbf{X}}$ and using equation (5) it is possible to calculate the quantities $\Delta \mathbf{X}$ and $\Delta \dot{\mathbf{X}}$. Subsequently, using the relations $\mathbf{X}_{k+1}=\mathbf{X}_{k}+\Delta \mathbf{X}$ and $\dot{\mathbf{X}}_{k+1}=\dot{\mathbf{X}}_{k}+\Delta \dot{\mathbf{X}}$ the 
1 quantities $\mathbf{X}_{k+1}$ and $\dot{\mathbf{X}}_{k+1}$ can be determined. To represent the solution in a matrix form, equation (9)

2 can be recast as:

$$
\Delta \ddot{\mathbf{X}}=\mathbf{B}^{-1} \Delta \mathbf{P}-\left[\Delta t \mathbf{B}^{-1} \mathbf{K}_{s}\right] \dot{\mathbf{X}}_{k}-\left[\Delta t \mathbf{B}^{-1} \mathbf{C}+\frac{1}{2}(\Delta t)^{2} \mathbf{B}^{-1} \mathbf{K}_{s}\right] \ddot{\mathbf{X}}_{k} ; \mathbf{B}=\mathbf{M}+\delta \Delta t \mathbf{C}+\alpha(\Delta t)^{2} \mathbf{K}{ }_{s}
$$

4 From equation (10), the acceleration $\ddot{\mathbf{X}}_{k+1}$ is given as:

$$
\ddot{\mathbf{X}}_{k+1}=\ddot{\mathbf{X}}_{k}+\Delta \ddot{\mathbf{X}}=\ddot{\mathbf{X}}_{k}+\mathbf{B}^{-1} \Delta \mathbf{P}-\left[\Delta t \mathbf{B}^{-1} \mathbf{K}_{s}\right] \dot{\mathbf{X}}_{k}-\left[\Delta t \mathbf{B}^{-1} \mathbf{C}+\frac{1}{2}(\Delta t)^{2} \mathbf{B}^{-1} \mathbf{K}_{s}\right] \ddot{\mathbf{X}}_{k}
$$

$$
=\mathbf{B}^{-1} \Delta \mathbf{P}-\left[\Delta t \mathbf{B}^{-1} \mathbf{K}_{s}\right] \dot{\mathbf{X}}_{k}+\left[\mathbf{I}-\Delta t \mathbf{B}^{-1} \mathbf{C}-\frac{1}{2}(\Delta t)^{2} \mathbf{B}^{-1} \mathbf{K}_{s}\right] \ddot{\mathbf{X}}_{k}
$$

Substituting equations (11) into equations (5) and making use of equation (3), we get:

$$
\left\{\begin{array}{l}
\mathbf{X}_{k+1} \\
\dot{\mathbf{X}}_{k+1} \\
\ddot{\mathbf{X}}_{k+1}
\end{array}\right\}=\mathbf{G}\left\{\begin{array}{l}
\mathbf{X}_{k} \\
\dot{\mathbf{X}}_{k} \\
\ddot{\mathbf{X}}_{k}
\end{array}\right\}+\mathbf{H} \Delta \mathbf{P} \text { or } \mathbf{q}\left(t_{k+1}\right)=\mathbf{G} \mathbf{q}\left(t_{k}\right)+\mathbf{H}\left[\ddot{x}_{g}\left(t_{k+1}\right)-\ddot{x}_{g}\left(t_{k}\right)\right]
$$

where,

$$
\mathbf{G}=\left[\begin{array}{ccc}
\mathbf{I} & (\Delta t) \mathbf{I}-\alpha(\Delta t)^{3} \mathbf{B}^{-1} \mathbf{K}_{s} & \frac{1}{2}(\Delta t)^{2} \mathbf{I}-\alpha(\Delta t)^{3} \mathbf{B}^{-1} \mathbf{C}-\frac{1}{2} \alpha(\Delta t)^{4} \mathbf{B}^{-1} \mathbf{K}_{s} \\
0 & \mathbf{I}-\delta(\Delta t)^{2} \mathbf{B}^{-1} \mathbf{K}_{s} & \Delta t \mathbf{I}-\delta(\Delta t)^{2} \mathbf{B}^{-1} \mathbf{C}-\frac{1}{2} \delta(\Delta t)^{3} \mathbf{B}^{-1} \mathbf{K}_{s} \\
0 & -\Delta t \mathbf{B}^{-1} \mathbf{K}_{s} & \mathbf{I}-\Delta t \mathbf{B}^{-1} \mathbf{C}-\frac{1}{2}(\Delta t)^{2} \mathbf{B}^{-1} \mathbf{K}_{s}
\end{array}\right] ; \mathbf{H}=-\left[\begin{array}{c}
\alpha(\Delta t)^{2} \mathbf{B}^{-1} \mathbf{M} \\
\delta \Delta t \mathbf{B}^{-1} \mathbf{M} \\
\mathbf{B}^{-1} \mathbf{M}
\end{array}\right]
$$

It may be emphasized that the matrices $\mathbf{K}_{\mathrm{s}}, \mathbf{G}$ and $\mathbf{H}$ are functions of time, and, thus are computed at each time step. The matrix $\mathbf{K}_{\mathrm{s}}$ is determined based on the deformed history of the structure. In the above formulation a viscous damping model is considered, and, thus the damping matrix $\mathbf{C}$ is constant. Hysteretic nonlinear damping can be also considered in which the damping matrix is treated as a variable and is computed at each time step.

It may be also emphasized that the stiffness used in the solution for time step $t_{k+1}$ is taken as the secant stiffness from time step $t_{k-1}$ to $t_{k}$. To correct this approximation, an iterative procedure for the stiffness $\mathbf{K}_{\mathrm{s}}$ is performed where the initial stiffness is replaced by $\mathbf{K}_{\mathrm{s}}^{(1)}, \mathbf{K}_{\mathrm{s}}^{(2)}, \ldots, \mathbf{K}_{\mathrm{s}}^{(\mathrm{n})}$ until a convergence criterion on $\mathbf{K}_{\mathrm{s}}$ is achieved. Finally, it may be also noted that the above formulation for inelastic response analysis is used as a subroutine to derive critical earthquake loads. The next section develops the modeling of critical earthquake loads for inelastic MDOF structures.

\section{Critical earthquake load inputs for MDOF inelastic structures}

The formulation for deriving critical earthquake excitations for MDOF inelastic structures is developed in this section. The ground acceleration appearing in the right side of equations (12) is represented as a product of a Fourier series and an envelope function:

$$
\ddot{x}_{g}(t)=e(t) \sum_{i=1}^{N_{f}} R_{i} \cos \left(\omega_{i} t-\varphi_{i}\right)
$$

Here, $R_{i}$ and $\varphi_{i}$ are $2 N_{f}$ unknown amplitudes and phase angles, respectively and $\omega_{i}, i=1,2, \ldots, N_{f}$ are the frequencies presented in the ground acceleration which are selected to span satisfactory the frequency range $\left(\omega_{0}, \omega_{c}\right)$. The envelope function $e(t)$ is taken as [18]:

$$
e(t)=A_{0}\left[\exp \left(-\alpha_{1} t\right)-\exp \left(-\alpha_{2} t\right)\right]
$$

where, $A_{0}$ is a scaling constant and the parameters $\alpha_{1}, \alpha_{2}$ impart the transient nature to $\ddot{x}_{g}(t)$. In constructing critical seismic inputs, the envelope function is taken to be known. Additionally, the information on energy $E$, peak ground acceleration (PGA) $M_{l}$, peak ground velocity (PGV) $M_{2}$, 
1 peak ground displacement (PGD) $M_{3}$, upper bound Fourier amplitude spectra (UBFS) $M_{4}(\omega)$ and 2 lower bound Fourier amplitude spectra (LBFS) $M_{5}(\omega)$ are also taken to be available which enables 3 the formulation of the following constraints:

$$
\begin{aligned}
& {\left[\int_{0}^{\infty} \ddot{x}_{g}^{2}(t) d t\right]^{\frac{1}{2}} \leq E} \\
& \max _{0<t<\infty}\left|\ddot{x}_{g}(t)\right| \leq M_{1} \\
& \max _{0<t<\infty}\left|\dot{x}_{g}(t)\right| \leq M_{2} \\
& \max _{0<t<\infty}\left|x_{g}(t)\right| \leq M_{3} \\
& M_{5}(\omega) \leq\left|X_{g}(\omega)\right| \leq M_{4}(\omega)
\end{aligned}
$$

Here, $X_{g}(\omega)$ is the Fourier transform of $\ddot{x}_{g}(t)$. It may be noted that the constraint on the earthquake energy $E$ is related to the Arias' intensity measure [19]. On the other hand, the constraints on upper and lower bound Fourier amplitude spectra are aimed to replicate the frequency content and amplitude of past recorded ground motion to the optimal earthquake acceleration. In other word, these constrains try to avoid the energy concentration of the ground acceleration at a narrow frequency range. To proceed further, the ground velocity and displacement are computed from equation (14) as follows:

$$
\begin{aligned}
& \dot{x}_{g}(t)=\sum_{i=1}^{N_{f}} R_{i} \int_{0}^{t} e(\tau) \cos \left(\omega_{i} \tau-\varphi_{i}\right) d \tau+C_{1} \\
& x_{g}(t)=\sum_{i=1}^{N_{f}} R_{i} \int_{0}^{t} e(\tau)(t-\tau) \cos \left(\omega_{i} t-\varphi_{i}\right) d \tau+C_{1} t+C_{2}
\end{aligned}
$$

The constants $C_{l}$ and $C_{2}$ are determined using the conditions $x_{g}(0)=0, \lim _{t \rightarrow \infty} \dot{x}_{g}(t) \rightarrow \infty$ [20], thus:

$$
C_{2}=0 ; C_{1}=-\sum_{i=1}^{N_{f}} R_{i} \int_{0}^{\infty} e(\tau) \cos \left(\omega_{i} \tau-\varphi_{i}\right) d \tau
$$

The constraints listed in equations (16) can be expressed in terms of the unknown variables $R_{i}, \varphi_{i}, i=1,2, \ldots, N_{f}$ as follows:

$$
\begin{aligned}
& {\left[A_{0}^{2} \sum_{m=1}^{N_{f}} \sum_{n=1}^{N_{f}} R_{m} R_{n} \int_{0}^{\infty}\left[\exp \left(-\alpha_{1} t\right)-\exp \left(-\alpha_{2} t\right)\right]^{2} \cos \left(\omega_{m} t-\varphi_{m}\right) \cos \left(\omega_{n} t-\varphi_{n}\right) d t\right]^{\frac{1}{2}} \leq E} \\
& \max _{0<t<\infty}\left|A_{0}\left[\exp \left(-\alpha_{1} t\right)-\exp \left(-\alpha_{2} t\right)\right] \sum_{n=1}^{N_{f}} R_{n} \cos \left(\omega_{n} t-\varphi_{n}\right)\right| \leq M_{1}
\end{aligned}
$$

$$
\begin{aligned}
& \max _{0<t<\infty} \mid A_{0} \sum_{n=1}^{N_{f}} \int_{0}^{t} R_{n}\left[\exp \left(-\alpha_{1} \tau\right)-\exp \left(-\alpha_{2} \tau\right)\right] \cos \left(\omega_{n} \tau-\varphi_{n}\right) d \tau- \\
& A_{0} \sum_{n=1}^{N_{f}} \int_{0}^{\infty} R_{n}\left[\exp \left(-\alpha_{1} \tau\right)-\exp \left(-\alpha_{2} \tau\right)\right] \cos \left(\omega_{n} \tau-\varphi_{n}\right) d \tau \mid \leq M_{2}
\end{aligned}
$$




$$
\begin{aligned}
& \max _{0<t<\infty} \mid A_{0} \sum_{n=1}^{N_{f}} \int_{0}^{t} R_{n}\left[\exp \left(-\alpha_{1} \tau\right)-\exp \left(-\alpha_{2} \tau\right)\right](t-\tau) \cos \left(\omega_{n} \tau-\varphi_{n}\right) d \tau- \\
& A_{0} t \sum_{n=1}^{N_{f}} \int_{0}^{\infty} R_{n}\left[\exp \left(-\alpha_{1} \tau\right)-\exp \left(-\alpha_{2} \tau\right)\right] \cos \left(\omega_{n} \tau-\varphi_{n}\right) d \tau \mid \leq M_{3} \\
& M_{5}(\omega) \leq\left|A_{0} \sum_{n=1}^{N_{f}} \int_{0}^{\infty} R_{n}\left\{\exp \left[-\left(\alpha_{1}+i \omega\right) t\right]-\exp \left[-\left(\alpha_{2}+i \omega\right) t\right]\right\} \cos \left(\omega_{n} t-\varphi_{n}\right) d t\right| \leq M_{4}(\omega) ; i=\sqrt{-1}
\end{aligned}
$$

2 To quantify the constraints quantities $E, M_{1}, M_{2}, M_{3}, M_{4}(\omega)$ and $M_{5}(\omega)$ it is assumed that a set of $N_{r}$ earthquake records denoted by $\ddot{v}_{g i}(t), i=1,2, \ldots, N_{r}$ are available for the site under

consideration or from other sites with similar geological soil conditions. The values of energy and peak values of acceleration, velocity and displacement are obtained for each of these records. The highest of these values across the ensemble of the records are taken to be the respective estimates of $E, M_{1}, M_{2}$ and $M_{3}$. The set of available records are further normalized such that the energy of each record is set to unity, and these normalized records are denoted by $\left\{\ddot{\bar{v}}_{g i}\right\}_{i=1}^{N_{r}}$. The bounds $M_{4}(\omega)$ and $M_{5}(\omega)$ are obtained as:

$$
M_{4}(\omega)=E \max _{1 \leq i \leq N_{r}}\left|\bar{V}_{g i}(\omega)\right| ; M_{5}(\omega)=E \min _{1 \leq i \leq N_{r}}\left|\bar{V}_{g i}(\omega)\right|
$$

The function $\bar{V}_{g i}(\omega), i=1,2, \ldots, N_{r}$ denotes the Fourier transform of the $i$ th normalized accelerogram $\ddot{v}_{g i}(t)$ which are computed using the Fast Fourier Transform. The idea of introducing an upper bound on the Fourier amplitude of the ground motion has been considered earlier [11,15,21,22]. The lower bound on the Fourier amplitude spectra was considered by Moustafa [4] and Abbas and Manohar [23]. Furthermore, the assumption on availability of past records is similar to the assumption made by Drenick [24] who employed past records as basis functions. In this paper, past records are used to quantify the constraints imposed on the critical ground motion.

It may be emphasized that the framework adopted for modeling the optimal earthquake ground motion in this paper belongs to the class of engineering models which do not explicitly account for the fault characteristics. The site soil condition, however, is automatically encapsulated in the set of records adopted in defining the constraints. Specifically, the constraint quantities $E, M_{1}, M_{2}, M_{3}$, $M_{4}(\omega)$ and $M_{5}(\omega)$ are quantified using past recorded earthquake data at the site or from other sites with similar geological soil conditions. This approach is consistent with the aspirations of the ground motion models that are commonly used by engineers, which, aim to replicate some of the gross features of recorded ground motions, such as, energy, amplitude, frequency content, nonstationarity trend, local soil amplification effects, and duration, see, e.g., [25-27]. As is well known, recorded ground accelerations contain the most useful information on earthquake ground motion [3]. It may be emphasized also that the engineering models are usually used when information on the source characteristics is either not available or limited. Seismological models, on the other hand, are analytical expressions that are based on attenuation relationships of ground motions which take into account several details, such as, fault dimension, fault orientation, rupture velocity, earthquake magnitude, stress drop, density of the intervening medium, local soil condition and source to site distance. These models have been developed in the literature, mainly by seismologists, see, e.g., [28-31]. To use these models, a host of parameters need to be defined and the success of the model depends on how these parameters are defined. Critical earthquake loads can be also formulated by using attenuation models in which the model parameters can be optimized to obtain the least favorable conditions. In this case, the class of admissible functions to be used in computing the optimal excitations becomes further constrained by the model adopted. The approach employed in this study, in this sense, is nonparametric in nature. 
Finally, the problem of deriving critical earthquake loads for inelastic structures can be posed as determining the optimization variables $y=\left\{R_{1}, R_{2}, \ldots, R_{N_{f}}, \varphi_{1}, \varphi_{2}, \ldots, \varphi_{N_{f}}\right\}^{t}$ such that the structure inelastic deformation is maximized subjected to the constraints listed in equations (19). The solution to this nonlinear constrained optimization problem is tackled by using the sequential quadratic programming method [32]. The optimization algorithm starts from an initial guess for the optimization variables $y$ and performs a sensitivity analysis for each iteration searching for new optimal values for $y$. The optimization converges to the optimal solution $y^{*}$ when convergence criteria on the objective function $f$ and on the optimization variables $y$ are satisfied, namely:

$$
\left|f_{j}-f_{j-1}\right| \leq \varepsilon_{1} ;\left|y_{i, j}-y_{i, j-1}\right| \leq \varepsilon_{2}
$$

Herein, $j$ is the iteration number, $i$ is the number of the optimization variable and $\varepsilon_{1}, \varepsilon_{2}$ are small quantities to be specified. The structure inelastic deformation is estimated by using the Newmark $\beta$-method as demonstrated in Section 2. The resulting nonlinear constrained optimization problem is tackled by using the sequential quadratic optimization algorithm 'fmincon' of the Matlab optimization toolbox [33].

The details of the procedure involved in the computation of the optimal earthquake and the associated inelastic deformation can be summarized as follows:

1. Define the structure parameters $\mathbf{M}, \mathbf{C}$ and $\mathbf{K}$, the initial yield displacement in tension and compression $\left(x_{y t}, x_{y c}\right)$ and associated yield strength.

2. Set the initial conditions $\mathbf{X}(0), \dot{\mathbf{X}}(0)$ and compute the initial acceleration $\ddot{\mathbf{X}}(0)$.

3. Perform free vibration analysis, select the time step $\Delta t$ and define the parameters of the Newmark $\beta$-method $(\delta, \alpha)$.

4. Specify initial guess for the optimization variables $y=\left[R_{1}, R_{2}, \ldots, R_{N_{f}}, \varphi_{1}, \varphi_{2}, \ldots, \varphi_{N_{f}}\right]^{t}$ and define $\ddot{x}_{g}(t)$.

5. Call the response analysis subroutine to calculate the structure's maximum response. This subroutine performs the following steps:

(a) At each point of time $t_{k}$ use the value of the parameter $\operatorname{KEY}\left(t_{k}\right)$ to establish the elastic or inelastic state of each member of the structure as follows:

- The $i$ th member behaves elastic when $\operatorname{KEY}(k, i)=0$.

- The $i$ th member behaves inelastic in tension when $\operatorname{KEY}(k, i)=1$.

- The $i$ th member behaves inelastic in compression when $\operatorname{KEY}(k, i)=-1$.

(b) Use the value of $\operatorname{KEY}\left(t_{k}\right)$ for each member to define the stiffness matrix $\mathbf{K}_{\mathrm{s}}$.

(c) Quantify the matrices $\mathbf{G}$ and $\mathbf{H}$ and evaluate the response $\mathbf{q}\left(t_{k}\right)$ using equation (12).

(d) Set the value for the parameter $\operatorname{KEY}(k+1, i)$ for each member at $t_{k}+1$ as follows:

- When the member is behaving elastic at the beginning of the time step $(\operatorname{KEY}(k, i)=0)$ then $\operatorname{KEY}(k+1, i)=0$ if $x_{y c}<x(k+1, i)<x_{y t}, \operatorname{KEY}(k+1, i)=1$ if $x(k+1, i)>x_{y t}$ and $\operatorname{KEY}(k+1, i)$ $=-1$ if $x(k+1, i)<x_{y c}$.

- When the member is behaving inelastic in tension at the beginning of the time step $(\operatorname{KEY}(k, i)=1)$ then $\operatorname{KEY}(k+1, i)=1$ if $\dot{x}(k+1, i)>0$ and $\operatorname{KEY}(k+1, i)=0$ if $\dot{x}(k+1, i)<0$.

- When the member is behaving inelastic in compression at the beginning of the time step $\operatorname{KEY}(k, i)=-1)$ then $\operatorname{KEY}(k+1, i)=-1$ if $\dot{x}(k+1, i)<0$ and $\operatorname{KEY}(k+1, i)=0$ if $\dot{x}(k+1, i)>0$.

6. Check if the convergence criteria of equation (21) are satisfied. If yes go to step 8 otherwise go to next step.

7. Call the Matlab optimization toolbox to generate new values for the optimization variable $y$ and return to step 5 . 
8. Recall the response analysis subroutine. This implies repeating steps (a)-(d) at all time points.

9. Compute the optimal solution as $f_{\max }^{*}=\max _{1 \leq j \leq N_{t}}\left|f_{\max }\left(t_{j}\right)\right|$ and the associated optimization variables $y^{*}=\left[A_{1}^{*}, A_{2}^{*}, \ldots, A_{N_{f}}^{*}, \varphi_{1}^{*}, \varphi_{2}^{*}, \ldots, \varphi_{N_{f}}^{*}\right]^{t}$.

It is to be noted that the inelastic response analysis routine is built as a subroutine inside the optimization program and is called at each iteration. Furthermore, the optimization is performed at discrete points of time and the optimal solution $y^{*}=\left[A_{1}^{*}, A_{2}^{*}, \ldots, A_{N_{f}}^{*}, \varphi_{1}^{*}, \varphi_{2}^{*}, \ldots, \varphi_{N_{f}}^{*}\right]^{t}$ is the one that produces the maximum objective function across all time points. The optimal earthquake loads are characterized in terms of the critical accelerations and associated inelastic deformation they produce. In this study, we also characterize critical inputs in terms of different energy forms dissipated by the structure. The next section demonstrates the quantification of various energy forms dissipated by inelastic structures.

\section{Energy dissipated by MDOF inelastic structures}

To gain more insights into the nature of optimal earthquake loads computed, different energy forms dissipated by the inelastic structure are quantified in this section. Several authors have characterized the structure response in terms of energy dissipated by the structure [34-36]. The input energy to the structure is dissipated by kinetic energy, damping energy and strain (elastic and plastic) energy. These energy terms can be quantified by integrating the structure equation of motion. Without loss of generality, consider an $N$-storey shear building frame. Thus, pre-multiplying equation (1) by $\dot{\mathbf{X}}^{T}$ and integrating we get:

$$
\int_{0}^{t} \dot{\mathbf{X}}^{T}(\tau) \mathbf{M} \ddot{\mathbf{X}}(\tau) d \tau+\int_{0}^{t} \dot{\mathbf{X}}^{T}(\tau) \mathbf{C} \dot{\mathbf{X}}(\tau) d \tau+\int_{0}^{t} \dot{\mathbf{X}}^{T}(\tau) \mathbf{F}_{s}(\tau) d \tau=-\int_{0}^{t} \dot{\mathbf{X}}^{T}(\tau) \mathbf{M}\{\mathbf{1}\} \ddot{x}_{g}(\tau) d \tau
$$

The right side of the above equation represents the input energy to the structure since ground starts shaking until it comes to rest. The first energy term of the left side is the relative kinetic energy $E_{\mathrm{K}}(t)$ of the masses associated with their motion relative to the ground and is given as:

$$
E_{K}(t)=\int_{0}^{t} \dot{\mathbf{X}}^{T}(\tau) \mathbf{M} \ddot{\mathbf{X}}(\tau) d \tau=\frac{1}{2} \sum_{i=1}^{N} m_{i} \dot{x}_{i}^{2}(t)
$$

The second energy term in equation (22) is the energy dissipated by damping $E_{\mathrm{D}}(t)$ given by:

$$
E_{D}(t)=\int_{0}^{t} \dot{\mathbf{X}}^{T}(\tau) \mathbf{C} \dot{\mathbf{X}}(\tau) d \tau=\sum_{i=1}^{N} \int_{0}^{t} \dot{x}_{i}(\tau) f_{D i}(\tau) d \tau
$$

For viscous damping models, the above expression reduces to $\sum_{i=1}^{N} \sum_{j=1}^{N} \int_{0}^{t} c_{i j} \dot{x}_{i}(\tau) \dot{x}_{j}(\tau) d \tau$. The third term of equation (22) is the sum of the recoverable strain energy $E_{\mathrm{s}}(t)$ and the hysteretic cumulative plastic energy dissipated by yielding, $E_{\mathrm{H}}(t)$ :

$$
E_{H}(t)=\sum_{i=1}^{N} \int_{0}^{t} \dot{x}_{i}(\tau) f_{s i}(\tau) d \tau-E_{s}(t)
$$

It may be noted that the recoverable strain energy and the kinetic energy vanish by the end of the earthquake duration and thus the input energy supplied to the structure by the earthquake ground motion is absorbed by the damping and the hysteretic mechanisms. In the present study, the time-variation of the above energy terms are employed in characterizing critical earthquake loads and associated structure deformation. The next section provides numerical illustrations for the formulation developed in this section and the previous two sections.

\section{Numerical results and discussions}

\subsection{Structure considered}


The two-story braced building frame shown in Figure 2 is considered to demonstrate the formulation developed in the previous sections. This structure was studied by Hart and Wong [17] for inelastic response analysis. The material behavior of braces 1 and 2 is taken as bilinear $\left(k_{2}=\gamma k_{1}\right)$ as shown in Figure 1(a). The mass and initial stiffness matrices of the structure are given as:

$$
\mathbf{M}=\left[\begin{array}{cc}
m_{1} & 0 \\
0 & m_{2}
\end{array}\right] ; \mathbf{K}_{e l}=\left[\begin{array}{cc}
\frac{A_{1} E}{L_{1}} \cos ^{2} \theta+\frac{A_{2} E}{L_{2}} \cos ^{2} \theta & -\frac{A_{2} E}{L_{2}} \cos ^{2} \theta \\
-\frac{A_{2} E}{L_{2}} \cos ^{2} \theta & \frac{A_{2} E}{L_{2}} \cos ^{2} \theta
\end{array}\right]
$$

The numerical values of floor masses are taken as $m_{1}=m_{2}=1.75 \times 10^{5} \mathrm{Ns}^{2} / \mathrm{m}$, the cross-sectional areas of the braces are $A_{1}=A_{2}=6.45 \times 10^{-4} \mathrm{~m}^{2}$, the Young's modulus $E=2.59 \times 10^{11} \mathrm{~N} / \mathrm{m}^{2}$, and the strain hardening ratio (i.e., ratio of the post-yield stiffness to the pre-yield stiffness) $\gamma=0.10$. When both braces are behaving elastically, the stiffness matrix $\mathbf{K}_{s}=\mathbf{K}_{e l}$, if brace 1 yields $\mathbf{K}_{s}=\mathbf{K}_{1}$, if brace 2 yields $\mathbf{K}_{s}=\mathbf{K}_{2}$ and if both braces yield $\mathbf{K}_{s}=\mathbf{K}_{12}$. These matrices are given as:

$$
\begin{aligned}
& \mathbf{K}_{e l}=\frac{A E}{L} \cos ^{2} \theta\left[\begin{array}{cc}
2 & -1 \\
-1 & 1
\end{array}\right] ; \mathbf{K}_{1}=\frac{A E}{L} \cos ^{2} \theta\left[\begin{array}{cc}
(1+\gamma) & -1 \\
-1 & 1
\end{array}\right] ; \\
& \mathbf{K}_{2}=\frac{A E}{L} \cos ^{2} \theta\left[\begin{array}{cc}
(1+\gamma) & -\gamma \\
-\gamma & \gamma
\end{array}\right] ; \mathbf{K}_{12}=\gamma \mathbf{K}_{e l}=\frac{A E}{L} \cos ^{2} \theta\left[\begin{array}{cc}
2 \gamma & -\gamma \\
-\gamma & \gamma
\end{array}\right]
\end{aligned}
$$

The structure is assumed to start from rest (i.e., $x_{1}=x_{2}=0, \dot{x}_{1}=\dot{x}_{2}=0$ ). The first two natural frequencies of the elastic structure were computed as $\omega_{1}=6.18 \mathrm{rad} / \mathrm{s}$ and $\omega_{2}=16.18 \mathrm{rad} / \mathrm{s}$. A Rayleigh proportional damping $\mathbf{C}=a \mathbf{M}+b \mathbf{K}_{s}$ with $a=0.2683, b=0.0027$ is adopted [16]. These values are selected such that the damping ratio in the first two modes is 0.03 . This implies that the damping forces in braces are nonlinear hysteretic functions of the deformed shape of the structure. Let the yield strain of the braces $\varepsilon_{y}=0.002$ for both tension and compression. The braces will yield at a relative displacement $x_{y}=L \varepsilon_{y} / \cos \theta=0.0381 \mathrm{~m}$. Thus, brace 1 yields when $\left|x_{1}\right|=0.0381 \mathrm{~m}$ and brace 2 yields when $\left|x_{2}-x_{1}\right|=0.0381 \mathrm{~m}$. The objective function is taken as the inter-story drift $x_{2}-x_{1}$. In the numerical analysis, the parameters of the Newmark $\beta$-method were taken as $\delta=1 / 2 ; \alpha=1 / 6$ and the time step $\Delta t=0.005 \mathrm{~s}$.

\subsection{Quantification of constraints}

A set of 20 earthquake ground motions $\left(N_{r}=20\right)$ is used to quantify the constraint limits $E, M_{1}, M_{2}$, $M_{3}, M_{4}(\omega)$ and $M_{5}(\omega)$ [37]. Table 1 summarizes relevant information on each of these records. These records include digitized information on ground acceleration, velocity and displacement and are measured on firm soil. Based on numerical analysis of these records the constraints were computed as $E=4.17 \mathrm{~m} / \mathrm{s}^{1.5}, M_{1}=4.63 \mathrm{~m} / \mathrm{s}^{2}(0.47 \mathrm{~g}), M_{2}=0.60 \mathrm{~m} / \mathrm{s}$ and $M_{3}=0.15 \mathrm{~m}$. The number of records $N_{r}=20$ was seen to produce considerably smooth upper and lower bounds on the Fourier coefficients of the ground acceleration. The average dominant frequency of the ground accelerations is seen to be around $1.64 \mathrm{~Hz}$. The envelope parameters were determined as $A_{0}=2.17$, $\alpha_{1}=0.13$, and $\alpha_{2}=0.50$. The convergence limits $\varepsilon_{1}, \varepsilon_{2}$ were taken as $10^{-6}$. The frequency content for $\ddot{x}_{g}(t)$ is taken as $(0.1-25) \mathrm{Hz}$. Additionally, in distributing the frequencies $\omega_{i}, i=1,2, \ldots, N_{f}$ in the interval $\left(\omega_{0}, \omega_{c}\right)$, (equation 14) it was found advantageous to select some of these $\omega_{i}$ to coincide exactly with the natural frequencies of the elastic structure and 
also to place relatively more points within the modal half-power bandwidth. The convergence criterion for the stiffness $\mathbf{K}_{\mathrm{s}}$ (Section 2) is taken as $10^{-3} \mathrm{~N} / \mathrm{m}$ and it was observed that $\mathbf{K}_{\mathrm{s}}$ converges within about 5 iterations.

As mentioned earlier, the resulting constrained nonlinear optimization problem is tackled by using the sequential quadratic optimization algorithm fmincon of the Matlab optimization toolbox [33]. This algorithm requires the specification of an initial guess for the vector of the optimization variables $y$. In the numerical calculations, alternative initial starting solutions, within the feasible region, were examined and it was found that all guesses lead to the same optimal solution. To select the number of frequency terms $N_{f}$ a parametric study was carried out and $N_{f}=51$ was found to give satisfactory results.

\subsection{Results and discussions}

The constraint scenarios considered in deriving critical earthquake inputs are listed in Table 2. The numerical results obtained are presented in figures 3-7 and Table 3 . The convergence of the objective function for case 4 is shown in figure 3(a). Figure 3(b) shows the inelastic inter-story drift normalized to the yield displacement. The time history of the optimal ground acceleration and associated Fourier amplitude spectrum for the earthquake load for case 1 is shown in Figure 4. Similar results for constraint scenarios 2 and 4 are shown in Figures 5 and 6, respectively. Figure 7 depicts the hysteretic restoring forces for braces 1 and 2. The hysteretic energy dissipated by yielding and damping (equations 23-25) are shown in Figure 8. Note that these quantities are computed using the horizontal displacements at the floors levels. Based on the extensive numerical results obtained, the following observations are made:

1. It is evident from the numerical results on critical ductility ratio and associated inelastic deformation response that the time variation of the structure deformation differs from that for the elastic structure. Unlike the elastic system, the inelastic system after it has yielded does not oscillate about its initial equilibrium position. Yielding causes the structure to drift from its initial equilibrium position and the system oscillates around a new equilibrium position until this gets shifted by another yielding. Accordingly, after the ground stops shaking, the structure comes to rest at a position different from its initial equilibrium position. In other words, the structure permanent deformation remains after ground stops shaking. For instance, the permanent displacement of the structure, for case $1, x_{p}=0.05 \mathrm{~m}$, and for case $4, x_{p}=0.02 \mathrm{~m}$.

2. The inelastic deformation, hysteretic energy dissipated by the structure and the frequency content of the critical earthquake are strongly dependent on the constraints imposed (Figures 4-6). If available information on the earthquake input is limited to energy and PGA, the critical input is highly resonant and response produced is conservative (Figure 4 and Table 3). It was observed that the Fourier amplitudes of the ground acceleration are resonant at two frequencies close to the elastic structure frequencies (slightly smaller than $\omega_{1}$ and $\omega_{2}$, see Figure 4). Additional constraints on UBFS and LBFS make the critical inputs realistic in terms of the frequency content $\left(\ddot{x}_{g}(t)\right.$ is rich in frequencies) and inelastic response they produce. Thus, the maximum ductility ratio $\mu$ for case 1 is 4.51 while that produced from constraint case 4 is 2.42 . Similarly, the maximum response reduces from $0.17 \mathrm{~m}$ to $0.09 \mathrm{~m}$ when the constraints on UBFS and LBFS are brought in. Additionally, the critical acceleration possesses a dominant frequency that is close to the average dominant frequency of past recorded ground motions. The constraints on energy, PGA, PGV and PGD (case 2) were not found to be significant in producing realistic critical inputs compared to the constraints on UBFS and LBFS, since the Fourier spectrum was overly conservative. For instance, while the optimization algorithm converges successfully to the optimal solution, the resulting Fourier spectrum of the ground acceleration were not seen to be realistic. In other words, the acceleration energy was concentrated at frequencies close to the 
natural frequencies of the elastic structure leading to ground motion that is poor in frequency content (see figure 5). Similar observations were also remarked for the ground velocity and displacement.

3. The earthquake input energy to the inelastic system is mainly dissipated by yielding and nonlinear damping of the structure (see Figure 8). The hysteretic and damping energies are significantly higher than the recoverable strain and kinetic energy. The kinetic and recoverable strain energies are small and diminish near the end of the ground shaking (Figure 8). The energy dissipated by yielding is significantly higher than that dissipated by damping (Figure 8(a)). It is observed also that the input energy to the inelastic system differs from that for the elastic system. The repeated yielding of the inelastic system indicates the level of the structure damage and the associated permanent deformation caused by the critical earthquake.

4. In deriving critical earthquake inputs for the inelastic structure, it was seen that cases 1 and 2 converge more rapidly compared to cases 3 and 4 . This is to be expected given that cases 1 and 2 contain 2 and 4 constraints, respectively. Cases 3 and 4, on the other hand, contain additional constraints on UBFS and LBFS which should be satisfied at discrete frequency points. It was also observed that the convergence rate of the objective function with respect to the number of iterations is faster for the elastic structure compared to that of the inelastic structure. Thus, for case 4 , the objective function for the linear case reaches initial convergence to the optimal solution within about 1,740 iterations, the corresponding number of iterations when inelastic behavior is considered is more than 9,000 . The final convergence of the objective function for the inelastic system is achieved within about 16,300 iterations (see figure 3(a)). It was, also, observed that the CPU time necessary for the convergence of the objective function for the inelastic system is about five times that for the elastic system.

To examine the effect of the strain hardening ratio $\left(\gamma=k_{2} / k_{1}\right)$ on the optimal earthquake acceleration computed, limited studies were carried out. The value of $\gamma$ was changed and the critical input was determined by solving a new optimization problem. Namely, $\gamma$ was taken as $0.10,0.05,0.01$ and 0 . The effect of the decrease in $\gamma$ was not seen to significantly influence the frequency content of the critical earthquake input. It was observed, however, that the inelastic structure with lower values of $\gamma$ yields more frequently compared to the same system with larger $\gamma$ values. The cumulative hysteretic energy dissipated, however, was observed to increase for higher values of $\gamma$ (Figure 8(b)). This feature is particularly remarkable at the end of the earthquake duration. It was also observed that results on critical earthquake accelerations from bilinear inelastic structure with $\gamma=0$ are similar to those for the elastic-plastic structure.

It was observed also that the inelastic structure with nonlinear Rayleigh damping dissipates more energy through damping mechanism compared to the same system with viscous damping. The influence of the variation of the damping ratio was also studied by varying this quantity. The damping ratio was taken as $0.01,0.03$ and 0.05 and solving a new optimization problem for each value. The effect of the damping ratio on the critical earthquake was not seen to significantly influence the frequency content of the earthquake acceleration. It was observed, however, that the ductility ratio for the structure increases for lower damping ratios. Thus, the ductility ratio decreases to 2.17 when the damping ratio is taken as 0.05 while the ductility ratio increases to 3.14 when the damping ratio is taken as 0.01 . It was also observed that inelastic structure with higher damping ratio dissipates more energy through damping.

As mentioned in the introduction section, the occurrence of earthquake ground motions involves a high level of uncertainty. In fact, each earthquake brings out new surprises and teaches us new lessons. The main objective beyond the use of the critical excitation method is to provide robust earthquake loads for seismic-resistant design of lifeline and important structures. The use of the method becomes of essential importance for structures that need to be constructed at seismic 
regions having limited earthquake data, and, also for structures that should remain functional after the earthquake occurrence (e.g., nuclear power plants, electrical facilities, water and chemicals storage tanks and hospitals). The earthquake loads derivable using this method are robust since these loads are tailored to produce the highest or the 'critical' structural responses (e.g., displacement, stresses, structural failure or damage) among all possible credible loads. Furthermore, the method provides an answer to the crucial question on the estimation of the worst possible scenario of the structure under seismic loads. In deed, the answer to this question represents one of the basic design concerns for structural engineers. Therefore, the method can be used by the structural engineer at preliminary design stages for prescribing robust earthquake loads on important and lifeline structures. The method can also be used for the seismic safety assessment of the structure by defining a failure criterion using a limit-state function, in terms of the structural capacity and associated quantity demanded by the critical earthquake, see, e.g., [13, 14]. Recent developments of the method include the inclusion of the nonlinear structural behavior $[13,15]$ and practical applications of the method to the seismic-resistant design of structures $[38,39]$. In these studies, the structure is designed (i.e. the cross-sectional dimensions of the structure are determined) iteratively such that it resists its dependent or variable critical earthquake load without failure. More recently, the critical excitation concept has been employed in identifying resonant ground motion records at a site, and, also in defining proper design earthquakes for structures [40, 41].

Finally, it may be noted that the framework adopted in this paper for modeling optimal earthquake loads on inelastic structures is deterministic in nature and does not provide information on the probability level associated with the design risk level. Hazard spectra in which earthquake loads are derived based on probability of occurrence or risk level, provide robust and powerful tool for modeling earthquake loads. This approach is of interest but was not considered in the present study.

\section{Concluding remarks}

The modeling of earthquake ground motions as design inputs for MDOF inelastic structures is studied. The earthquake acceleration is expanded in terms of a Fourier series modulated by an envelope function. The coefficients of the series representation are estimated such that the normalized inelastic inter-story drift is maximized subjected to predefined constraints. These constraints are taken to reflect known characteristics of recorded ground motions such as the earthquake energy, upper and lower bounds on the Fourier spectra of the ground acceleration. The constraints, also, contain upper limits on PGA, PGV and PGD. The framework adopted in deriving earthquake loads belongs to the class of engineering models which do not account for source characteristics but aims to replicate gross features of recorded ground motions. The material force-displacement behavior is modeled using hysteretic bilinear and elastic-plastic laws. The resulting nonlinear optimization problem is solved by using the sequential quadratic optimization method.

It is shown that critical earthquake loads for the inelastic structure differ from those for the elastic structure. Similarly, the time variation of the structure deformation differs from those of the elastic system. Unlike the elastic system, the inelastic system dissipates energy through yielding and damping. The present study, also, examined the modeling of damping using nonlinear Rayleigh model. The effect of variations in the damping ratio and also in the strain hardening ratio on the derived critical acceleration and associated optimal inelastic deformation were also studied.

The proposed formulation was demonstrated with reference to the seismic inelastic response analysis of a two-storey building frame. Given the complexity of engineering structures it is of interest to examine the formulation developed in this paper for more complex structures. This can be achieved by combining nonlinear optimization techniques with nonlinear finite element software. 
This work was partly supported by the Japanese Society for the Promotion of Science. The support is gratefully acknowledged. The author is also thankful for two anonymous reviewers for careful reading of the paper and useful comments made.

\section{References}

[1] R.W. Clough, J. Penzien, Dynamics of structures, Chapter 24: Seismological background, Computers \& Structures Inc., 3rd edition, CA, 2003.

[2] T. Kusky, Earthquakes: Plate tectonics and earthquake hazards, Facts on file Inc., NY, 2008.

[3] J.J. Bommer, A.B. Acevedo, The use of real earthquake accelerograms as input to dynamic analysis, Journal of Earthquake Engineering 1 (2004) 43-91.

[4] A. Moustafa, Deterministic/Reliability-based critical earthquake load models for linear/nonlinear engineering structures, Ph. D. thesis, Department of Civil Engineering, Indian Institute of Science, 2002.

[5] I. Takewaki, Seismic critical excitation method for robust design: A review, Journal of Structural Engineering 128 (2002) 665-672.

[6] R.N. Iyengar, Worst inputs and a bound on the highest peak statistics of a class of non-linear systems, Journal of Sound and Vibration 25 (1972) 29-37.

[7] R.F. Drenick, The critical excitation of nonlinear systems, Journal of Applied Mechanics 18 (1977) 333-336.

[8] A.J. Philippacopoulos, P.C. Wang, Seismic inputs for nonlinear structures, Journal of Engineering Mechanics 110 (1984) 828-836.

[9] B.D. Westermo, The critical excitation and response of simple dynamic systems, Journal of Sound and Vibration 100 (1985) 233-242.

[10] A.A. Pirasteh, J.L. Cherry, J.A. Balling, The use of optimization to construct critical accelerograms for given structures and sites, Earthquake Engineering \& Structural Dynamics 16 (1988) 597-613.

[11] Takewaki, Probabilistic critical excitation for MDOF elastic-plastic structures on compliant ground, Earthquake Engineering \& Structural Dynamics 30 (2001) 1345-1360.

[12] Takewaki, Critical excitation for elastic-plastic structures via equivalent linearization, Probabilistic Engineering Mechanics 17 (2002) 73-84.

[13] A.M. Abbas, C.S. Manohar, Reliability-based critical earthquake load models. Part 2: Nonlinear structures, Journal of Sound and Vibration 287 (2005) 883-900.

[14] A.M. Abbas, C.S. Manohar, Reliability-based vector nonstationary random critical earthquake excitations for parametrically excited systems, Structural Safety 29 (2007) 32-48.

[15] A.M. Abbas, Critical seismic load inputs for simple inelastic structures, Journal of Sound and Vibration 296 (2006) 949-967.

[16] A.K. Chopra, Dynamics of structures, 3rd edition, Prentice Hall, NJ, 2006.

[17] G.C. Hart, K. Wong, Structural dynamics for structural engineers, John Wiley \& Sons, NY, 2000.

[18] M. Shinozuka, Y. Sato, Simulation of nonstationary random process, Journal of Engineering Mechanics 91 (1967) 11-40.

[19] A. Arias, A measure of earthquake intensity: Seismic design of nuclear power plants, Cambridge, MA, MIT press, 1970, 438-468.

[20] M. Shinozuka, L. Henry, Random vibration of a beam column, Journal of Engineering Mechanics 91 (1965) 123-143. 
[21] M. Shinozuka, Maximum structural response to seismic excitations, Journal of Engineering Mechanics 96 (1970) 729-738.

[22] A. Baratta, I. Elishakoff, G. Zuccaro, M. Shinozuka, A generalization of the Drenick-Shinozuka model for bounds on the seismic response of a single-degree-of-freedom system, Earthquake Engineering \& Structural Dynamics 27 (1998) 423-437.

[23] A.M. Abbas, C.S. Manohar, Investigations into critical earthquake load models within deterministic and probabilistic frameworks, Earthquake Engineering \& Structural Dynamics 31 (2002) 813-832.

[24] R.F. Drenick, Aseismic design by way of critical excitation, Journal of Engineering Mechanics 99 (1973) 649-667.

[25] A. Der Kiureghian, J. Crempien, An evolutionary model for earthquake ground motion, Structural Safety 6 (1989) 235-246.

[26] J.P. Conte, B.F. Peng, Fully nonstationary analytical earthquake ground-motion model. Journal of Engineering Mechanics 123(1) (1997) 15-24.

[27] W.-L. He, A.K. Agrawal, Analytical model of ground motion pulses for the design and assessment of seismic protective systems, Journal of Structural Engineering 134 (2008) 1177-88.

[28] D.M. Boore, Stochastic simulation of high-frequency ground motions based on seismological models of the radiated spectra, Bulletin of Seismological Society of America 73(1983) 1865-94.

[29] J.N. Brune, Tectonic stress and the spectra of seismic shear waves from earthquakes, Journal of Geophysics Research 75 (1970) 4997-5009.

[30] T.G. Hanks, R.K. McGuire, The character of high frequency ground motions based on seismic shear waves, Bulletin of Seismological Society of America 71(1981) 2071-95.

[31] S.T. Quek, Y.P. Teo, T. Balendra, Non-stationary structural response with evolutionary spectra using seismological input model, Earthquake Engineering \& Structural Dynamics 19(1990) $275-88$

[32] J.S. Arora, Introduction to optimum design, Elsevier Academic Press, San Diego, 2004.

[33] T. Caleman, M.A. Branch, A. Grace, Optimization toolbox for the use with Matlab, user's guide version 2(Release 11)\}, The Math Works Inc., USA, 1999.

[34] T.F. Zahrah, W.J. Hall, Earthquake energy absorbtion in sdof structures, Journal of Structural Engineering 110 (1984) 1757-1772.

[35] H. Akiyama, Earthquake-resistant limit-state design for buildings, University of Tokyo Press, Tokyo, 1985.

[36] C.M. Uang, V.V. Bereto, Evaluation of seismic energy in structures, Earthquake Engineering \& Structural Dynamics 19 (1990) 77-90.

[37] Consortium organizations for strong-motion observation systems, "http://db.cosmos-eq.org/scripts/default.plx", 2005.

[38] I. Takewaki, Robust building stiffness design for variable critical excitations, Journal of Structural Engineering 128 (2002) 1565-1574.

[39] S. Saha, C.S. Manohar, Inverse reliability based structural design for system dependent critical earthquake loads, Probabilistic Engineering Mechanics 20 (2005) 19-31.

[40] A. Moustafa, Discussion on a new approach of selecting real input ground motions for seismic design: The most unfavorable real seismic design ground motions, Earthquake Engineering \& Structural Dynamics, in press (DOI: 10.1002/eqe.885).

[41] A. Moustafa, Damage-based design earthquake loads for SDOF inelastic structures, Journal of Structural Engineering, under review. 
Table 1

Information on past recorded ground motions for a firm soil site [37]

\begin{tabular}{|c|c|c|c|c|c|c|c|c|}
\hline Earthquake & $\begin{array}{l}\text { Richter } \\
\text { Magnitude }\end{array}$ & $\begin{array}{c}\text { Source-site } \\
\text { distance }(\mathrm{km})\end{array}$ & Component & $\begin{array}{l}\text { PGA } \\
\left(\mathrm{m} / \mathrm{s}^{2}\right)\end{array}$ & $\begin{array}{l}\mathrm{PGV} \\
(\mathrm{m} / \mathrm{s})\end{array}$ & $\begin{array}{l}\text { PGD } \\
(\mathrm{m})\end{array}$ & $\begin{array}{l}\text { Energy }^{*} \\
\left(\mathrm{~m} / \mathrm{s}^{1.5}\right)\end{array}$ & $\begin{array}{l}\text { Recording } \\
\text { station site }\end{array}$ \\
\hline Mammoth lakes & 6.2 & 1.5 & $\mathrm{~W}$ & 4.02 & 0.21 & 0.05 & 3.73 & \multirow{2}{*}{$\begin{array}{l}\text { Convict } \\
\text { Greek }\end{array}$} \\
\hline 05.25 .1980 & & & S & 3.92 & 0.23 & 0.05 & 4.01 & \\
\hline Loma prieta & \multirow[t]{2}{*}{7.0} & \multirow[t]{2}{*}{9.7} & $\mathrm{~W}$ & 3.91 & 0.31 & 0.07 & 3.82 & \multirow[t]{2}{*}{ Capitola } \\
\hline 10.18 .1989 & & & S & 4.63 & 0.36 & 0.11 & 2.61 & \\
\hline Morgan hill & \multirow[t]{2}{*}{6.1} & \multirow[t]{2}{*}{4.5} & S60E & 3.06 & 0.40 & 0.07 & 2.33 & \multirow[t]{2}{*}{ Halls Valley } \\
\hline 04.24 .1971 & & & S30W & 1.53 & 0.30 & 0.02 & 1.64 & \\
\hline San Fernando & \multirow[t]{2}{*}{6.6} & \multirow[t]{2}{*}{27.6} & N69W & 3.09 & 0.17 & 0.04 & 2.07 & \multirow{2}{*}{$\begin{array}{l}\text { Castaic Old } \\
\text { ridge }\end{array}$} \\
\hline 02.09 .1971 & & & $\mathrm{~N} 21 \mathrm{E}$ & 2.66 & 0.28 & 0.10 & 2.47 & \\
\hline Parkfield & \multirow[t]{2}{*}{5.0} & \multirow[t]{2}{*}{9.1} & $\mathrm{~W}$ & 2.88 & 0.44 & 0.01 & 1.33 & \multirow{2}{*}{$\begin{array}{l}\text { Parkfield } \\
\text { Fault }\end{array}$} \\
\hline 12.20 .1994 & & & S & 3.80 & 0.10 & 0.01 & 1.74 & \\
\hline Caolinga & \multirow[t]{2}{*}{6.5} & \multirow[t]{2}{*}{30.1} & $\mathrm{~W}$ & 2.83 & 0.26 & 0.10 & 2.67 & \multirow{2}{*}{$\begin{array}{l}\text { Cantua } \\
\text { Creek }\end{array}$} \\
\hline 05.02 .1983 & & & $\mathrm{~N}$ & 2.20 & 0.26 & 0.10 & 2.14 & \\
\hline Northridge & \multirow[t]{2}{*}{6.7} & \multirow[t]{2}{*}{5.9} & S74E & 3.81 & 0.60 & 0.12 & 4.17 & \multirow[t]{2}{*}{ Canoga Park } \\
\hline 01.17.1994 & & & S16W & 3.43 & 0.34 & 0.09 & 3.50 & \\
\hline Cape Mendocino & \multirow[t]{2}{*}{7.0} & \multirow[t]{2}{*}{5.4} & $\mathrm{~W}$ & 3.25 & 0.45 & 0.15 & 2.44 & \multirow{2}{*}{$\begin{array}{l}\text { Petrolia } \\
\text { general }\end{array}$} \\
\hline 04.25 .1992 & & & S & 2.89 & 0.24 & 0.08 & 2.31 & \\
\hline Westmorland & \multirow[t]{2}{*}{5.0} & \multirow[t]{2}{*}{6.6} & $\mathrm{E}$ & 4.35 & 0.33 & 0.11 & 3.26 & \multirow{2}{*}{$\begin{array}{l}\text { Westmor } \\
\text { land fire }\end{array}$} \\
\hline 04.26 .1981 & & & $\mathrm{~S}$ & 3.54 & 0.44 & 0.15 & 3.25 & \\
\hline Imperial valley & \multirow[t]{2}{*}{6.4} & \multirow[t]{2}{*}{17.4} & $\mathrm{~S} 45 \mathrm{~W}$ & 2.68 & 0.22 & 0.10 & 2.30 & \multirow{2}{*}{$\begin{array}{l}\text { Calexico } \\
\text { Fire }\end{array}$} \\
\hline 10.15 .1979 & & & $\mathrm{~N} 45 \mathrm{~W}$ & 1.98 & 0.19 & 0.15 & 2.14 & \\
\hline
\end{tabular}

* Note that the energy is estimates as $E=\left[\int_{0}^{\infty} \ddot{x}_{g}^{2}(t) d t\right]^{1 / 2}$. 
Table 2

Nomenclature of constraint scenarios considered

\begin{tabular}{cl}
\hline Case & Constraints imposed \\
\hline 1 & Energy and PGA \\
2 & Energy, PGA, PGV and PGD \\
3 & Energy, PGA and UBFS \\
4 & Energy, PGA, UBFS and LBFS \\
\hline
\end{tabular}

Table 3

Inelastic deformation quantities for alternative constraints $(\gamma=0.10, \zeta=0.03)$

\begin{tabular}{llll}
\hline Case & $\Delta x_{\max }(\mathrm{m})$ & $\mu_{\max }$ & $x_{p}(\mathrm{~m})$ \\
\hline 1 & 0.17 & 4.51 & 0.05 \\
2 & 0.14 & 3.66 & 0.03 \\
3 & 0.11 & 2.91 & 0.03 \\
4 & 0.09 & 2.42 & 0.02 \\
\hline
\end{tabular}

Note that $\Delta x_{\max }=$ maximum inter-story drift, $\mu_{\max }=$ maximum absolute ductility ratio and $x_{p}=$ permanent deformation. 


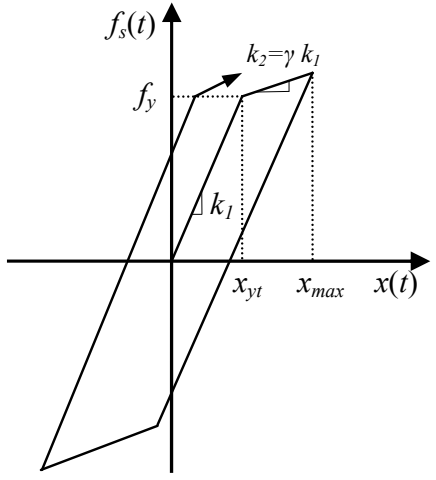

(a) Bilinear model

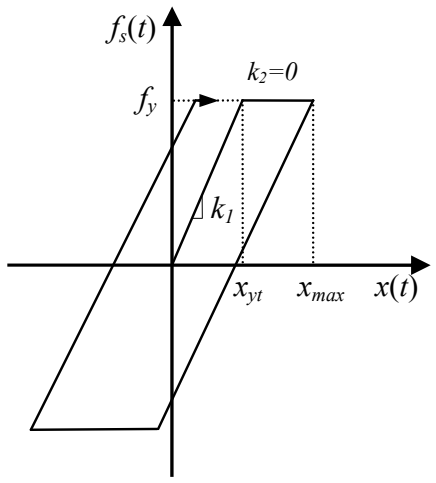

(b) Elastic-plastic

Fig. 1. (a) Force-displacement relation for nonlinear materials. 


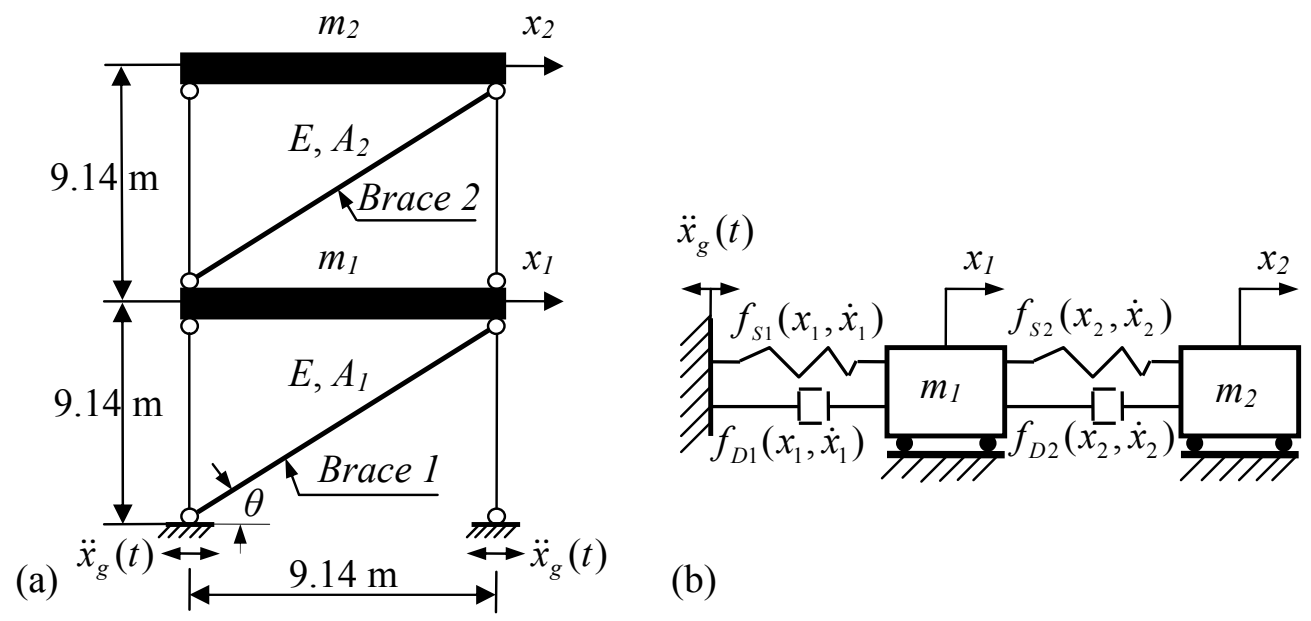

Fig. 2. (a) Two-story inelastic building frame (b) Mechanical system. 

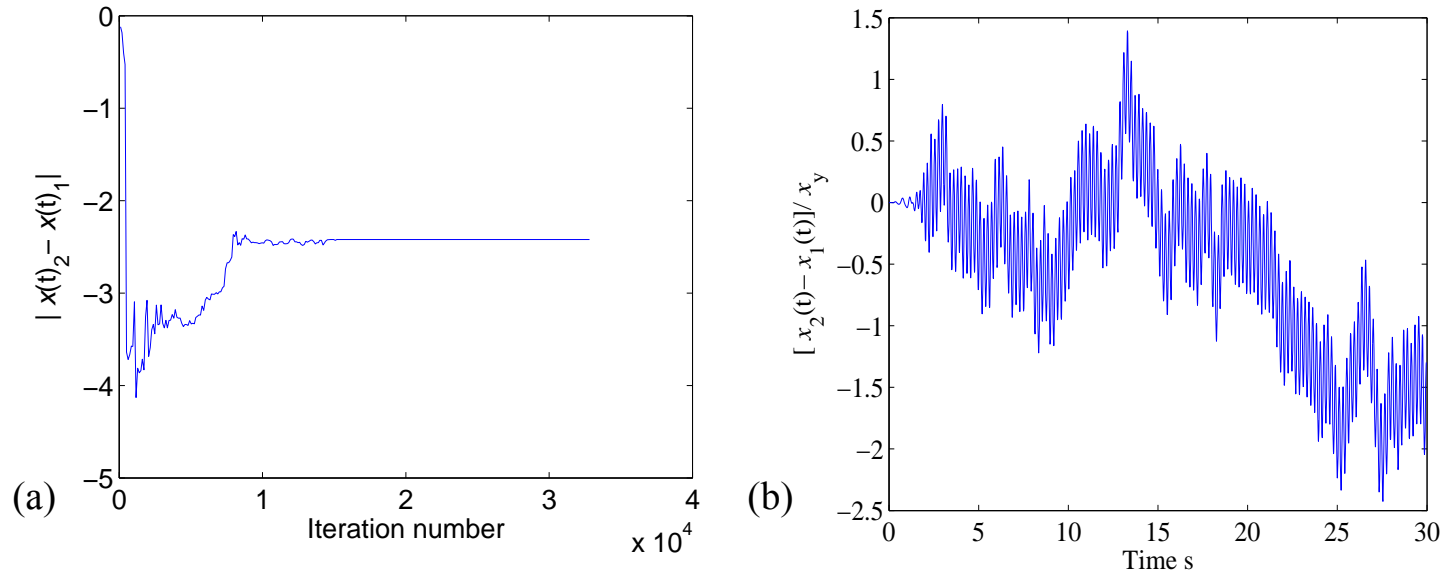

Fig. 3. (a) Convergence of objective function (b) Normalized inter-story drift. 

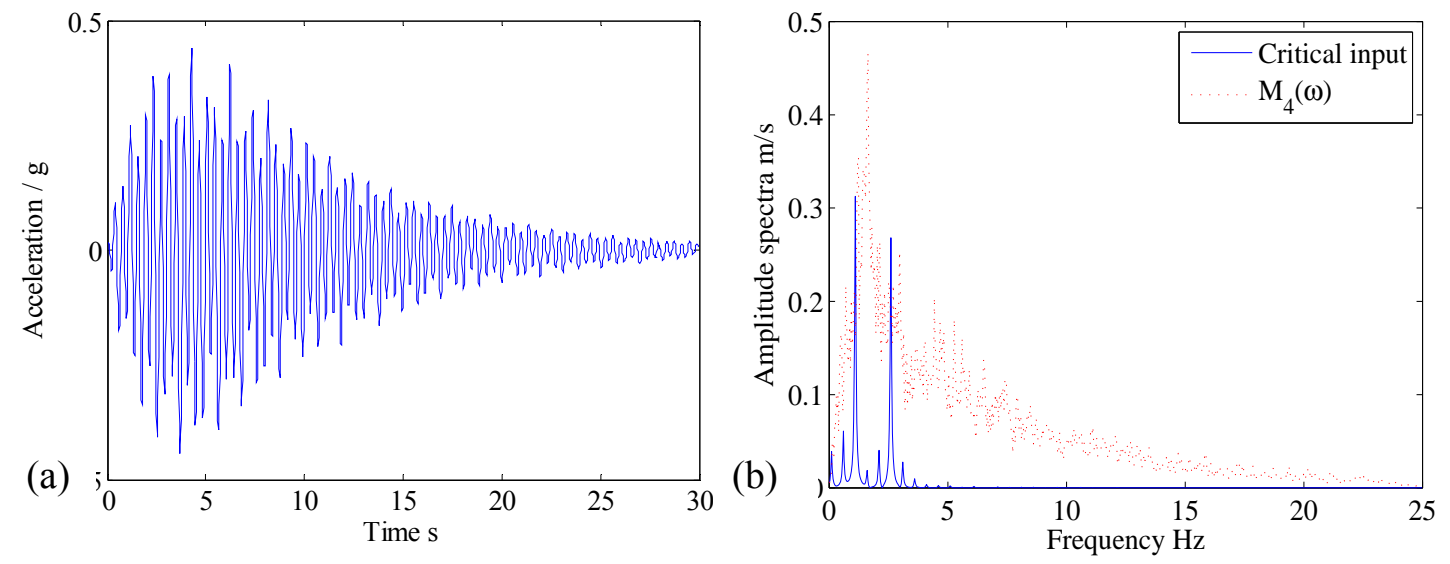

Fig. 4. Critical acceleration $\ddot{x}_{g}(t)$ for inelastic structure for case 1 (a) Time history (b) Fourier amplitude spectrum. 

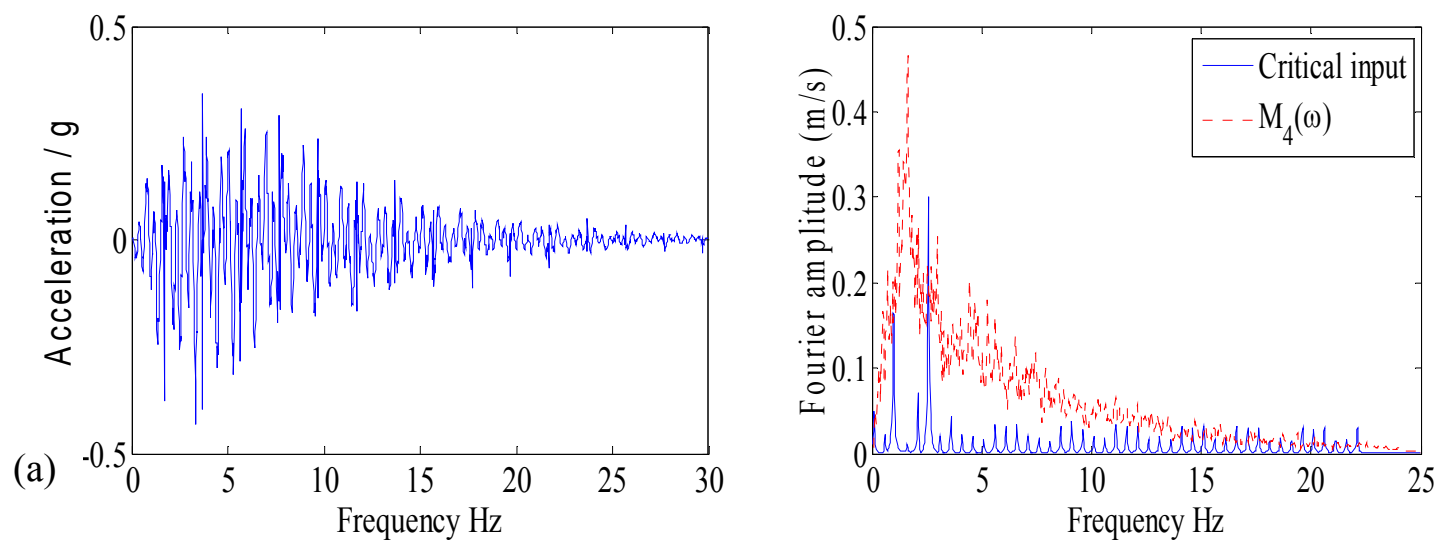

Fig. 5. Critical acceleration $\ddot{x}_{g}(t)$ for inelastic structure for case 2 (a) Time history (b) Fourier amplitude spectrum. 

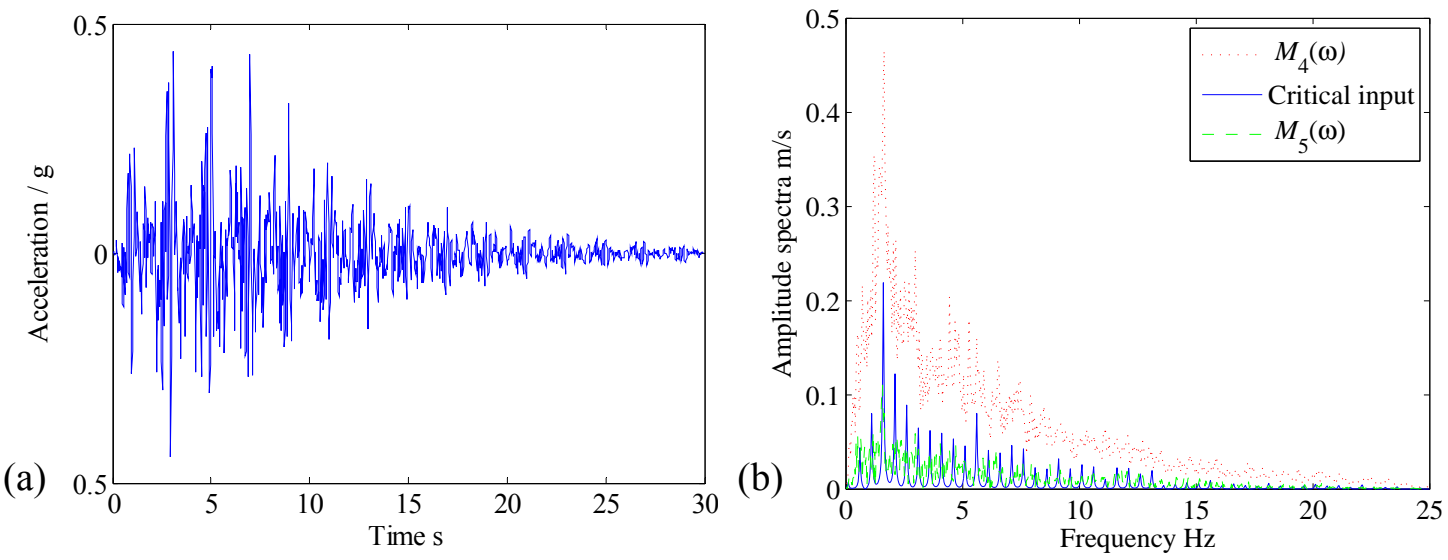

Fig. 6. Critical acceleration $\ddot{x}_{g}(t)$ for inelastic structure for case 4 (a) Time history (b) Fourier amplitude spectrum. 

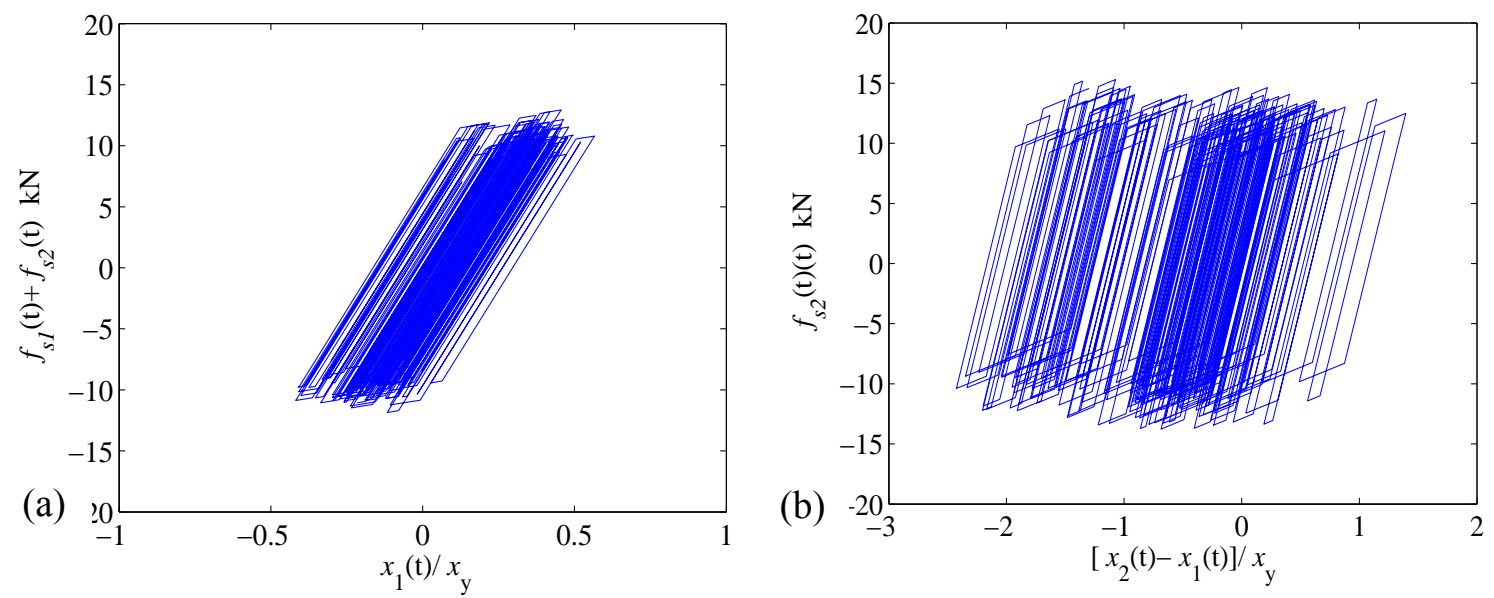

Fig. 7. Hysteretic restoring force for the inelastic structure for case 4. 

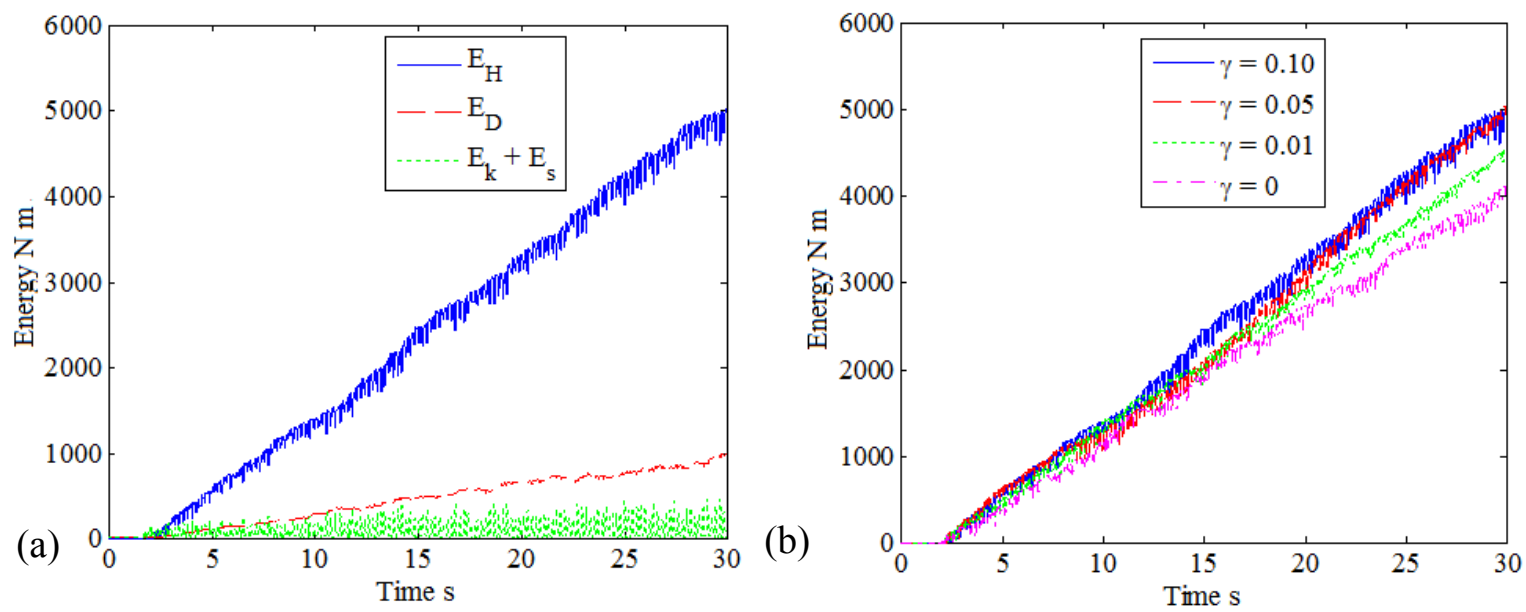

Fig. 8. (a) Various energies dissipated by the structure $(\gamma=0.10, \zeta=0.03)$ (b) Hysteretic cumulative energy $(\zeta=0.03)$. 